\title{
An algorithm for calculating critical probabilities and percolation functions in percolation models defined by rotations
}

\author{
RONALD W. J. MEESTER \\ Faculty of Technical Mathematics and Informatics, Delft University of Technology, \\ Julianalaan 132, 2628 BL Delft, The Netherlands
}

(Received 29 July 1988 and revised 13 February 1989)

\begin{abstract}
A class of percolation models on $\mathbb{Z}^{d}$ is developed in which the stochastic structure is provided by means of a $d$-parameter dynamical system. Of particular interest are those models generated by circle rotations. Unlike for independent models, the critical value and the percolation function can be explicitly calculated. These calculations lead to a conjecture concerning the behaviour of a related dynamical system.
\end{abstract}

\section{Introduction}

Percolation theory-in particular critical values and percolation functions-has been an object for mathematical study for the last three decades. Until now, mainly independent models have been studied. The calculation of critical values in such models turns out to be very difficult. Only in some very special, symmetric models, for example independent bond percolation on the square-or triangular lattice, the critical value is known today. (See [Ka].) In other models, such as independent site percolation on the square lattice, very little can be said rigorously about the critical value. (See e.g. $[\mathrm{B}],[\mathrm{T}]$.)

As far as the percolation function is concerned, it seems yet to be impossible to calculate the percolation function with any precision. In fact, one of the main problems in percolation theory concerns the behaviour of this function near the critical value. (See e.g. [Kb], [Kc].)

In this paper we investigate some parametric dependent percolation models and in particular we show that for circle and interval percolation (to be defined in $\$ 3$ ), the critical value and the percolation function can be explicitly calculated. These calculations lead to a conjecture concerning the behaviour of a dynamical system. The models we discuss appear as special cases of a general model of which independent site percolation on $\mathbb{Z}^{d}$ is another special case. 
In $\S 2$ we formulate the model and prove some general results. In $\$ 3$ we give some examples of the model, including circle and interval percolation. In $\$ 4$ we show how to calculate the critical value in these models. The final section is devoted to an investigation of the percolation function.

\section{The model and some general properties}

Let $(\Omega, \mathscr{A}, \mu)$ be a non-atomic measure space. Let $d \geq 2$ and let $f$ be an action of $\mathbb{Z}^{d}$ on $\Omega$, i.e. $f$ is a measurable map from $\mathbb{Z}^{d} \times \Omega$ to $\Omega$ with the properties that (i) $f(0, \omega)=\omega$ where 0 denotes the null vector, and (ii) $f\left(z, f\left(z^{\prime}, \omega\right)\right)=f\left(z+z^{\prime}, \omega\right)$, for every $z, z^{\prime} \in \mathbb{Z}^{d}$ and every $\omega \in \Omega$. If $z \in \mathbb{Z}^{d}$ and $\omega \in \Omega$ then we write for simplicity $z(\omega):=f(z, \omega)$. It is clear that the map $z$ is invertible and that $z^{-1} \equiv-z$, for every $z \in \mathbb{Z}^{d}$. In this paper we assume that for all $z \in \mathbb{Z}^{d}$ the map $z$ is measure-preserving. Of course, $f$ is completely determined by the commuting maps $e_{1}, \ldots, e_{d}: \Omega \rightarrow \Omega$, where $e_{1}, \ldots, e_{d}$ denote the unit vectors of $\mathbb{Z}^{d}$. We say that $f$ is aperiodic if $\mu\{\omega \mid z(\omega)=\omega\}=0$, for every $z \in \mathbb{Z}^{d} \backslash\{0\}$.

A path in $\mathbb{Z}^{d}$ is an infinite sequence $\pi=\left(\pi_{0}, \pi_{1}, \ldots\right)$ such that $\pi_{i} \in \mathbb{Z}^{d}$ for every $i \in \mathbb{N}, \pi_{0}=0$ and $d\left(\pi_{i}, \pi_{i+1}\right)=1$, for every $i \in \mathbb{N}$, where $d$ denotes Euclidean distance. A circuit is a finite subsequence $\left(\pi_{i}, \pi_{i+1}, \ldots, \pi_{j}\right)$ of $\pi$ such that $\pi_{i}=\pi_{j}$. A path is called self-avoiding if it contains no circuits. Let $\Pi_{d}$ be the set of all self-avoiding paths in $\mathbb{Z}^{d}$. For any path $\pi$, define $\tilde{\pi}=\left(\tilde{\pi}_{1}, \tilde{\pi}_{2}, \ldots\right):=\left(\pi_{1}-\pi_{0}, \pi_{2}-\pi_{1}, \pi_{3}-\pi_{2}, \ldots\right)$. Then $\tilde{\pi} \in\left\{ \pm e_{1}, \ldots, \pm e_{d}\right\}^{N}$. It is clear that $\pi \in \prod_{d}$ iff $\sum_{j=n}^{m} \tilde{\pi}_{j} \neq 0$, for every $n<m \in \mathbb{N}$. In such a case $\tilde{\pi}$ will also be said to be self-avoiding. We will need the following concept associated with self-avoiding paths. Let $\pi$ and $\pi^{\prime}$ be self-avoiding paths. For a fixed $n \in \mathbb{N}$, consider the path $\pi^{*}$, defined by

$$
\tilde{\pi}^{*}=\left(\tilde{\pi}_{1}, \ldots, \tilde{\pi}_{n}, \tilde{\pi}_{1}^{\prime}, \tilde{\pi}_{2}^{\prime}, \ldots\right) \text {. }
$$

$\pi^{*}$ is not necessarily self-avoiding. However, $\pi^{\prime}$ is self-avoiding and therefore $\pi^{*}$ only contains finitely many circuits. Let $\left(\pi_{i}^{*}, \ldots, \pi_{j}^{*}\right)$ be such a circuit. Consider the path $\left(\pi_{0}^{*}, \ldots, \pi_{i}^{*}, \pi_{j+1}^{*}, \ldots\right)$ which is the original path with this circuit removed. The path obtained from $\pi^{*}$ by removing all circuits in this way is self-avoiding and is called the $n$-concatenation of $\pi$ and $\pi^{\prime}$.

For $\pi \in \prod_{d}$, define $\pi^{l}:=\left(\pi_{0}, \ldots, \pi_{l}\right)$, for every $l \in \mathbb{N}$. Let $\prod_{d}^{l}$ be the set $\left\{\pi^{l} \mid \pi \in \prod_{d}\right\}$. Now we come to our main definition.

Definition 2.1. For $A \in \mathscr{A}$ the set

$$
\mathscr{P}(A):=\left\{\omega \in A \mid \text { there is a } \pi \in \prod_{d} \text { such that } \pi_{i}(\omega) \in A, \text { for all } i \in \mathbb{N}\right\}
$$

is called the percolation set of $A$. If $\omega$ and $\pi$ are as in this definition, we say that $\omega$ percolates in $A$, along $\pi$.

We think of $\mathscr{P}(A)$ as the subset of $A$ containing exactly those elements $\omega$ for which it is possible to select a sequence of maps $\tilde{\pi} \in\left\{ \pm e_{1}, \ldots, \pm e_{d}\right\}^{N}$ which is self-avoiding, and such that $\tilde{\pi}_{1} \cdots \tilde{\pi}_{n}(\omega) \in A$ for every $n \in \mathbb{N}$.

Proposition 2.2. For $A \in \mathscr{A}$, the percolation set $\mathscr{P}(A)$ is measurable.

Proof. For $\pi^{l} \in \prod_{d}^{l}$, let $A\left(\pi^{l}\right):=\left\{\omega \mid \pi_{1}(\omega) \in A, \ldots, \pi_{l}(\omega) \in A\right\}$. Clearly, $A\left(\pi^{l}\right) \in \varnothing$ 
for all $\pi^{l}$. We claim that

$$
\mathscr{P}(A)=A^{*}:=\bigcap_{l=1}^{\infty} \bigcup_{\pi^{\prime} \in \prod_{d}^{\prime}} A\left(\pi^{l}\right),
$$

which suffices to prove the proposition. The inclusion $\mathscr{P}(A) \subset A^{*}$ is trivial. To prove the reverse inclusion, suppose $\omega \in A^{*}$. Then there exists a sequence $\left(\pi^{(1)}, \pi^{(2)}, \ldots\right)$ such that $\pi^{(i)} \in \prod_{d}^{i}$ and $\pi_{n}^{(i)}(\omega) \in A$, for all $n \leq i$. But $\pi_{1}^{(i)}$ can, viewed as elements of $\mathbb{Z}^{d}$, only take $2 d$ values. Therefore we can find a $\pi_{1} \in \mathbb{Z}^{d}$ and a subsequence $\left(k_{1}, k_{2}, \ldots\right)$ of $(1,2, \ldots)$ such that $\pi_{1}^{\left(k_{i}\right)}=\pi_{1}$, for every $i \in \mathbb{N}$. Now there again is at least one $\pi_{2}$ such that $\pi_{2}^{\left(k_{i}\right)}=\pi_{2}$ for infinitely many $i \in \mathbb{N}$. Proceeding in this way, we define $\pi:=\left(\pi_{0}, \pi_{1}, \ldots\right)$, where $\pi_{0}=0$. It is clear that $\pi \in \prod_{d}$ and that $\omega$ percolates in $A$ along $\pi$ so $\omega \in \mathscr{P}(A)$.

Definition 2.3. $A \in \mathscr{A}$ percolates if $\mu(\mathscr{P}(A))>0$.

The following theorem and its proof were suggested by M. Keane. The theorem shows that the collection of percolating sets of $\mathscr{A}$ is in a certain sense irregular.

THEOREM 2.4. Consider the model described above. Suppose in addition that $f$ is aperiodic and that $\mu(\Omega)=1$. Then, for every $\varepsilon>0$ there exists a set $A_{\varepsilon} \in \mathscr{A}$ with $\mu\left(A_{\varepsilon}\right)>1-\varepsilon$ which does not percolate. Furthermore there exists a set $B_{\varepsilon} \in \mathscr{A}$ with $\mu\left(B_{\varepsilon}\right)<\varepsilon$ which does percolate.

Proof. The proof is based on the following generalization to $\mathbb{Z}^{d}$ of the well known Rokhlin-Lemma. A proof can be found in [C].

A box $\Lambda=\Lambda\left(r_{1}, \ldots, r_{d}\right)$ in $\mathbb{Z}^{d}$ is defined as

$$
\Lambda=\left\{z \in \mathbb{Z}^{d} \mid 0 \leq z_{i} \leq r_{i}, \quad 1 \leq i \leq d\right\} .
$$

The interior $\Lambda^{0}$ of $\Lambda\left(r_{1}, \ldots, r_{d}\right)$ is defined as

$$
\Lambda^{0}\left(r_{1}, \ldots, r_{d}\right)=\left\{z \in \mathbb{Z}^{d} \mid 0<z_{i}<r_{i}, \quad 1 \leq i \leq d\right\} .
$$

Under the conditions mentioned above, for each $\delta>0$ and for each box $\Lambda$ in $\mathbb{Z}^{d}$, there exists a set $C=C_{\delta, \Lambda} \in \mathscr{A}$ such that $z, z^{\prime} \in \Lambda, z \neq z^{\prime}$ implies $z(C) \cap z^{\prime}(C)=\varnothing$ and

$$
\mu\left(\bigcup_{z \in \Lambda} z(C)\right)>1-\delta \text {. }
$$

Using this result, fix $\varepsilon>0$ and choose $\Lambda$ and $\delta$ such that

$$
\mu\left(\bigcup_{z \in \Lambda^{0}} z\left(C_{\delta, \Lambda}\right)\right)>1-\varepsilon
$$

It is easy to see that this choice is always possible. Now define the following sets ( $C$ denotes $C_{\delta, \Lambda}$ for our particular choice of $\delta$ and $\Lambda$ ):

$$
\begin{aligned}
& A_{\varepsilon}:=\bigcup_{z \in \Lambda^{0}} z(C), \\
& B_{\varepsilon}:=A_{\varepsilon}^{c} .
\end{aligned}
$$

We claim that $\mathscr{P}\left(A_{\varepsilon}\right)=\varnothing$ and that $\mu\left(\mathscr{P}\left(B_{\varepsilon}\right)\right)>0$, which suffices to prove the theorem. To see this, suppose $\omega \in \mathscr{P}\left(A_{\varepsilon}\right)$ percolates along $\pi$. Let $z_{j}$ be defined by $\pi_{j}(\omega) \in z_{j}(C)$. 
Then

$$
\begin{aligned}
\pi_{j+1}(\omega) & =\left(\pi_{j+1}-\pi_{j}\right)\left(\pi_{j}(\omega)\right) \in\left(\pi_{j+1}-\pi_{j}\right)\left(z_{j}(C)\right) \\
& =\left(z_{j}+\pi_{j+1}-\pi_{j}\right)(C) .
\end{aligned}
$$

On the other hand, $\pi_{j+1}(\omega) \in z_{j+1}(C)$ by the definition of $z_{j+1}$. If $z_{j} \in \Lambda^{0}$, then $\left(z_{j}+\pi_{j+1}-\pi_{j}\right) \in \Lambda$ and it follows from the disjointness of the $z(C)$ 's that $z_{j}+\pi_{j+1}-\pi_{j}=z_{j+1}$, for all $j \geq 0$. By finiteness of $\Lambda^{0}$, there exist $i, i^{\prime}$ such that $z_{i}=z_{i^{\prime}}$ and hence $\pi_{i}=\pi_{i}$, so $\pi$ contains a circuit which is a contradiction.

To prove the second statement, fix $\omega \in B_{\varepsilon}$. If $e_{1}(\omega) \notin B_{\varepsilon}$, then $e_{1}(\omega) \in z^{\prime}(C)$ for some $z^{\prime} \in \Lambda^{0}$ and hence $\omega \in z(C)$ where $z=z^{\prime}-e_{1} \in \Lambda \backslash \Lambda^{0}$. But $z+e_{1} \in \Lambda^{0}$ and hence $z+e_{2} \in \Lambda \backslash \Lambda^{0}$. It follows that $e_{2}(\omega) \in\left(z+e_{2}\right)(C) \subset B_{\varepsilon}$. This means that for all $\omega \in B_{\varepsilon}$, either $e_{1}(\omega) \in B_{\varepsilon}$ or $e_{2}(\omega) \in B_{\varepsilon}$. This implies that $\mathscr{P}\left(B_{\varepsilon}\right)=B_{\varepsilon}$ and hence $B_{\varepsilon}$ percolates.

Now let us consider the model from the following point of view. Given $\omega \in \Omega$ and $A \in \mathscr{A}$, we say that an element $z \in \mathbb{Z}^{d}$ is open if $z(\omega) \in A$. If $z(\omega) \notin A$, $z$ is closed. To any element $(\omega, A) \in \Omega \times \mathscr{A}$ corresponds a configuration of $\mathbb{Z}^{d}$ in which all sites are either open or closed. An open path is a path whose elements are all open. It is easily seen that $A$ percolates iff

$\mu\{\omega \mid(\omega, A)$ corresponds to a configuration

that contains an open, self-avoiding path $\}>0$.

Our model can therefore be seen as a generalization of site percolation in $\mathbb{Z}^{d}$.

The preceding theorem shows that under general conditions there exist arbitrary small sets which percolate, and arbitrary large ones which do not. In standard percolation theory however, percolation occurs if the probability for a site to be open, independent of all other sites, exceeds a certain value, called the critical value. Analogously, in our model we would like percolation to occur if sets become sufficiently large. This can be achieved when additional structure is imposed on our model. To restrict ourselves to a one-dimensional subset of $\mathscr{A}$, we make the following definition.

Definition 2.5. Let $\mathrm{I} \subset \mathbb{R}$ be an interval of the form $[0, r], 0 \leq r \leq \infty$. A parametrization in $\mathscr{A}$ is a map $h: I \rightarrow \mathscr{A}$, given by $h(p)=A_{p}$ such that $\mu\left(A_{0}\right)=0, A_{p} \subset A_{q}$ for all $0 \leq p \leq q \leq r$.

It is clear that $\mathscr{P}\left(A_{p}\right) \subset \mathscr{P}\left(A_{q}\right)$ for all $0 \leq p \leq q \leq r$. Consequently, the following definition makes sense.

Definition 2.6. (i) Given a parametrization in $\mathscr{A}$, the percolation function, denoted by $\Theta$, is defined as

$$
\Theta(p)=\mu\left(\mathscr{P}\left(A_{p}\right)\right), \quad p \in I .
$$

(ii) The critical value, denoted by $p_{c}$, is defined as

$$
p_{c}=\inf \{p \in I \mid \Theta(p)>0\}
$$

In the examples in the following sections, we need more structure on the space $\Omega$ as well. Therefore we state the following. 
Proposition 2.7. Suppose $\Omega$ is a metric space, $\mathscr{A}$ is the Borel $\sigma$-field and $f$ is a continuous action of $\mathbb{Z}^{d}$ on $\Omega$. Then $\mathscr{P}(A)$ is closed if $A$ is closed.

Proof. Using (*) in the proof of Proposition 2.2, $\mathscr{P}(A)$ is a countable intersection of closed sets and hence closed.

\section{Some examples}

In this section we discuss three examples of our model.

3.1. Independent site percolation. Let $\Omega$ be the set $[0,1]^{\mathbf{Z}^{2}}, \mathscr{A}=\mathscr{B}^{\mathrm{Z}^{2}}$ where $\mathscr{B}$ denotes the Borel $\sigma$-field on the unit interval, and let $\mu=\lambda^{\mathbb{Z}^{2}}$, where $\lambda$ denotes Lebesguemeasure on $[0,1]$. Let $f$ be given by $\left(e_{1}(\omega)\right)_{i, j}=\omega_{i+1, j},\left(e_{2}(\omega)\right)_{i, j}=\omega_{i, j+1}$, for all $\omega \in \Omega$. Define a parametrization $h^{\prime}$ by

$$
h^{\prime}(p)=\left\{\omega \in \Omega \mid \omega_{0,0} \leq p\right\}, \quad 0 \leq p \leq 1 .
$$

Then we have a model for ordinary independent site percolation on the square-lattice. The critical value is unknown in this case. (See e.g. [B], [T].)

3.2. Interval percolation. Let $\Omega=\mathbb{R}, \mathscr{A}$ the Borel $\sigma$-field and $\mu$ denote Lebesgue measure. (Note that Theorem 2.4 may not be valid in this case, as the measure is infinite.) Define $e_{1}, \ldots, e_{d}: \Omega \rightarrow \Omega$ as

$$
e_{i}(\omega)=\omega+\alpha_{i}, \quad 1 \leq i \leq d,
$$

for some $0 \leq \alpha_{1} \leq \cdots \leq \alpha_{d}$. Define a parametrization $h$ by $h(p)=[0, p], p \geq 0$. The critical value in this case will be denoted by $p_{c}^{l}\left(\alpha_{1}, \ldots, \alpha_{d}\right)$ or just by $p_{c}^{I}$ if no confusion is possible. Similarly, we write $\Theta^{I}\left(\alpha_{1}, \ldots, \alpha_{d}\right)$ or $\Theta^{I}$ for the percolation function in this case. The set $\mathscr{P}\left(A_{p}\right)=\mathscr{P}([0, p])$ will be denoted by $\mathscr{P}_{p}^{I}\left(\alpha_{1}, \ldots, \alpha_{d}\right)$ or $\mathscr{P}_{p}^{I}$.

3.3. Circle percolation. Let $\Omega$ be the space $\mathbb{R} / \mathbb{Z}$. Let $\mu$ and $\mathscr{A}$ be as in example 3.2.

Define $f$ by $e_{i}(\omega)=\omega+\alpha_{i}(\bmod 1), 1 \leq i \leq d$, where we assume without loss of generality that $0 \leq \alpha_{1} \leq \cdots \leq \alpha_{d} \leq \frac{1}{2}$. Let a parametrization $g$ be given by $g(p)=$ $[0, p], 0 \leq p<1$. The critical value will be denoted by $p_{c}^{C}=p_{c}^{C}\left(\alpha_{1}, \ldots, \alpha_{d}\right)$, the percolation function by $\Theta^{C}=\Theta^{C}\left(\alpha_{1}, \ldots, \alpha_{d}\right)$. The set $\mathscr{P}\left(A_{p}\right)=\mathscr{P}([0, p])$ will be denoted by $\mathscr{P}_{p}^{C}\left(\alpha_{1}, \ldots, \alpha_{d}\right)$ or $\mathscr{P}_{p}^{C}$.

We shall analyze the last two examples in detail in the following sections.

In the first place we are interested in circle percolation because this is a stationary model for site percolation in $\mathbb{Z}^{d}$. Interval percolation turns out to be useful in the analysis of circle percolation.

4. The critical values $p_{c}^{I}$ and $p_{c}^{C}$

We start with some easy preliminaries, which will turn out to be useful. If we write $\mathscr{P}_{p}, p_{c}$ or $\Theta$ without superscript, this will always mean $\mathscr{P}_{p}^{I}, p_{c}^{I}$ resp. $\Theta^{I}$.

LeMma 4.1. Consider interval percolation. Then we have

(i) if $\mathscr{P}_{p} \neq \varnothing$ for some $p>0$, then $0 \in \mathscr{P}_{p}$ and $p \in \mathscr{P}_{p}$.

(ii) $p_{c}=\inf \left\{p \mid \mathscr{P}_{p} \neq \varnothing\right\}$.

(iii) Let $p_{c, \omega}:=\inf \left\{p \mid \omega \in \mathscr{P}_{p}\right\}$, then $p_{c}=p_{c, 0}$.

Analogous statements are valid for circle percolation. 
Proof. To prove (i), let $\omega_{0}:=\inf \left\{\omega \mid \omega \in \mathscr{P}_{p}\right\}$. It follows from Proposition 2.7 that $\omega_{0} \in \mathscr{P}_{p}$. Suppose $\omega_{0}$ percolates in $A_{p}$ along $\pi$. It is clear from the choice of $\omega_{0}$ that $\pi_{n}\left(\omega_{0}\right) \geq \omega_{0}$, for all $n$. This implies by translation that $0 \leq \pi_{n}(0) \leq p-\omega_{0}$, for all $n \in \mathbb{N}$, and it follows that $\omega_{0}=0$. By a symmetric argument we see that $p \in \mathscr{P}_{p}$. To prove (ii), let $p_{c}^{\prime}:=\inf \left\{p \mid \mathscr{P}_{p} \neq \varnothing\right\}$. We have $p_{c} \geq p_{c}^{\prime}$ trivially. For the reverse inequality, suppose $\mathscr{P}_{p} \neq \varnothing$ for some $p>0$. Fix $\varepsilon>0$. According to (i), $0 \in \mathscr{P}_{p}$. Suppose 0 percolates in $A_{p}$ along $\pi$. Then $\pi_{n}(\omega) \in[\omega, p+\omega]$, for all $\omega$, and this implies that $[0, \varepsilon] \subset \mathscr{P}_{p+\varepsilon}$, so $\mu\left(\mathscr{P}_{p+\varepsilon}\right)>0$. Since $\varepsilon$ is arbitrary, this proves $p_{c} \leq p_{c}^{\prime}$. Of course (iii) immediately follows from (i) and (ii).

It is clear that we can use an analogous proof in the case of circle percolation.

To study the relation between interval and circle percolation, we make the following convention, which is valid until the statement of Theorem 4.5.

Elements of $\mathbb{Z}^{d}$ correspond with maps from $\mathbb{R} / \mathbb{Z}$ to $\mathbb{R} / \mathbb{Z}$ as in circle percolation with parameters $\alpha_{1}, \ldots, \alpha_{d}$. Elements of $\mathbb{Z}^{d^{\prime}}$, with $2 d \geq d^{\prime}>d$ correspond with maps from $\mathbb{R}$ to $\mathbb{R}$ as in interval percolation with parameters $\alpha_{1}, \ldots, \alpha_{d}, 1-\alpha_{d}, \ldots$, $1-\alpha_{2 d-d^{\prime}+1}$.

Let $e_{i}, i=1, \ldots, d$ denote the unit vectors in $\mathbb{Z}^{d}$, and let $e_{i}^{1}, e_{i}^{2}, i=1, \ldots, d$ denote the unit vectors in $\mathbb{Z}^{2 d}$. Now fix $\omega \in \mathbb{R} / \mathbb{Z}$ and let $\pi \in \prod_{d}$, the set of self-avoiding paths in $\mathbb{Z}^{d}$. Observe that $e_{i}(\omega)$ is equal to either $\omega+\alpha_{i}$ or $\omega-\left(1-\alpha_{i}\right)$. Write $e_{i}^{1}(\omega)$ for $\omega+\alpha_{i}$ and $e_{i}^{2}(\omega)$ for $\omega-\left(1-\alpha_{i}\right)$. $e_{i}^{j}$ maps $\mathbb{R}$ into $\mathbb{R}$ and a sequence of maps $\left(e_{i_{1}}^{j_{1}}, e_{i_{2}}^{j_{2}}, \ldots\right)$ can be identified with a path in $\mathbb{Z}^{2 d}$. So with $\omega$ fixed, we have a 1-1-correspondence between all paths $\pi$ in $\mathbb{Z}^{d}$ and the set of all paths $\pi^{\sigma}$ in $\mathbb{Z}^{2 d}$ with the property that $0 \leq \pi_{n}^{\sigma}(\omega)<1$. To study this correspondence in detail, we state the following lemmas.

LEMMA 4.2. Let $\pi$ be a path in $\mathbb{Z}^{d}$ and let $\pi^{\sigma}$ in $\mathbb{Z}^{2 d}$ be its corresponding path, with some fixed $\omega \in \Omega$. Suppose in addition that $\tilde{\pi}_{i}^{\sigma} \in\left\{ \pm e_{1}^{1}, \pm e_{2}^{1}, \ldots, \pm e_{d}^{1}, \pm e_{d}^{2}\right\}$, for all $i$. Then $\pi$ is self-avoiding iff $\pi^{\sigma}$ is self-avoiding.

Proof. It is immediate that $\sum_{n=i}^{j} \tilde{\pi}_{n}^{\sigma}=0$ implies $\sum_{n=i}^{j} \tilde{\pi}_{n}=0$, for all $i, j$ with $i<j$. For the reverse, fix $i$ and $j$ and let $n_{ \pm k}:=\operatorname{card}\left\{i \leq n \leq j \mid \tilde{\pi}_{n}^{\sigma}= \pm e_{k}^{1}\right\}, k=1, \ldots, d$ and let $n_{ \pm(d+1)}:=$ card $\left\{i \leq n \leq j \mid \tilde{\pi}_{n}^{\sigma}= \pm e_{d}^{2}\right\}$. Suppose $\sum_{n=i}^{j} \tilde{\pi}_{n}=0$. This implies that $\pi_{j}^{\sigma}(\omega)-\pi_{i-1}^{\sigma}(\omega)=n_{-(d+1)}-n_{d+1}$. If this last expression is not zero it must be at least one in absolute value, but this is impossible because $0 \leq \pi_{n}^{\sigma}(\omega)<1$, for all $n$. So we have $n_{d+1}=n_{-(d+1)}$ and it follows that $n_{k}=n_{-k}$ for all $1 \leq k \leq d+1$ and the lemma follows.

The following lemma compares two interval percolation problems with different parameter sets.

LEMMA 4.3. Consider interval percolation and let $0 \leq \alpha_{1} \leq \cdots \leq \alpha_{d} \leq \frac{1}{2}$. Then

(i) $p_{c}\left(\alpha_{1}, \alpha_{2}, \ldots, \alpha_{d}, 1-\alpha_{d}\right) \leq 1-\alpha_{d-1}$.

(ii) $\mathscr{P}_{p}\left(\alpha_{1}, \ldots, \alpha_{d}, 1-\alpha_{d}, \ldots, 1-\alpha_{1}\right)=\mathscr{P}_{p}\left(\alpha_{1}, \alpha_{2}, \ldots, \alpha_{d}, 1-\alpha_{d}\right)$.

(iii) $p_{c}\left(\alpha_{1}, \ldots, \alpha_{d}, 1-\alpha_{d}, \ldots, 1-\alpha_{1}\right)=p_{c}\left(\alpha_{1}, \alpha_{2}, \ldots, \alpha_{d}, 1-\alpha_{d}\right)$. 
Proof. (i) We will construct a $\pi \in \prod_{d+1}$ with $0 \leq \pi_{n}(0) \leq 1-\alpha_{d-1}$, for all $n \in \mathbb{N}$. The inequality in (i) then follows from an application of Lemma 4.1(iii).

If $\alpha_{d}=\alpha_{d-1}$, then we take the path $\pi$ defined by $\tilde{\pi}=\left(e_{d},-e_{d-1}, e_{d},-e_{d-1}, \ldots\right)$.

If $\alpha_{d} \neq \alpha_{d-1}$, we construct $\pi$ as follows. Start in 0 and consider the sequence of alternating $\alpha_{d}$ and $-\alpha_{d-1}$ until we reach the interval $\left[1-\alpha_{d}, 1-\alpha_{d-1}\right]$. Then the next element of the sequence is $-\left(1-\alpha_{d}\right)$. After a step $-\left(1-\alpha_{d}\right)$ we end up at a value $\delta$, say, in the interval $\left[0, \alpha_{d}-\alpha_{d-1}\right]$. Now the construction works, starting from $\delta$, just as well as starting from 0 . This means that we can repeat this construction infinitely many times. The path constructed this way has the desired property and is self-avoiding.

(ii) We need only to prove that the 1.h.s. is contained in the r.h.s. as the other inclusion is trivial. Suppose $\omega \in$ l.h.s. and suppose that $\omega$ percolates in $A_{p}$ along $\pi$. Consider the first index $i_{0}$, say, such that $\tilde{\pi}_{i_{0}} \notin\left\{ \pm e_{1}^{1}, \pm e_{2}^{1}, \ldots, \pm e_{d}^{1}, \pm e_{d}^{2}\right\}$, and suppose $\tilde{\pi}_{i_{0}}$ has a plus sign. Let $\pi^{0} \in \prod_{d+1}$ be such that $0 \leq \pi_{n}^{0}(0) \leq 1-\alpha_{d-1}$, for all $n \in \mathbb{N}$. According to (i), $\pi^{0}$ exists. Now consider the $\left(i_{0}-1\right)$-concatenation $\pi^{\prime}$, say, of $\pi$ and $\pi^{0}$. It is clear that $\pi^{\prime}$ has the following property:

$$
0 \leq \inf _{n} \pi_{n}^{\prime}(\omega) \leq \sup _{n} \pi_{n}^{\prime}(\omega) \leq \sup _{n} \pi_{n}(\omega) \text {. }
$$

If $\tilde{\pi}_{i_{0}}$ has a minus sign, we replace 0 by $p_{c}$. Because $\pi^{\prime} \in \prod_{d+1}$, this proves (ii).

(iii) This immediately follows from (ii).

Now we are ready to prove that circle percolation is in fact a special case of interval percolation.

THEOREM 4.4. Circle percolation with parameters $0 \leq \alpha_{1} \leq \cdots \leq \alpha_{d} \leq \frac{1}{2}$ is equivalent to interval percolation with parameters $\alpha_{1}, \ldots, \alpha_{d}, 1-\alpha_{d}$ in the sense that

$$
\mathscr{P}_{p}^{C}\left(\alpha_{1}, \ldots, \alpha_{d}\right)=\mathscr{P P}_{p}^{I}\left(\alpha_{1}, \alpha_{2}, \ldots, \alpha_{d}, 1-\alpha_{d}\right), \quad 0 \leq p<1 .
$$

In the l.h.s. we take, with a slight abuse of notation, the representatives of the equivalence classes of $\mathbb{R} / \mathbb{Z}$ which are contained in the interval $[0,1)$.

Proof. Suppose $\omega \in$ l.h.s. and let $\omega$ percolate in $A_{p}$ along $\pi$. According to Lemma 4.3 we may assume that $\tilde{\pi}_{i}^{\sigma} \in\left\{ \pm e_{1}^{1}, \pm e_{2}^{1}, \ldots, \pm e_{d}^{1}, \pm e_{d}^{2}\right\}$, for all $i$. But $\pi$ is selfavoiding and Lemma 4.2 implies that $\pi^{\sigma}$ is self-avoiding as well. This implies that $\omega \in$ r.h.s. The reverse inclusion is proved analogously.

The preceding theorem shows that as far as $p_{c}^{C}$ is concerned, we can restrict ourselves to interval percolation. We will analyze interval percolation in detail and we begin with the two-dimensional case. (The convention made after Lemma 4.1 ends here.)

THEOREM 4.5. Suppose $0<\alpha_{1} \leq \alpha_{2}$ are given. If $\alpha_{1} / \alpha_{2} \notin \mathbb{Q}$ then

$$
p_{c}\left(\alpha_{1}, \alpha_{2}\right)=\alpha_{1}+\alpha_{2} \text {. }
$$

If $m_{1} \alpha_{1}=m_{2} \alpha_{2}$ for some $m_{1}, m_{2} \in \mathbb{N}$ relatively prime, then

$$
p_{c}\left(\alpha_{1}, \alpha_{2}\right)=\alpha_{2}+\frac{m_{2}-1}{m_{2}} \alpha_{1}=\frac{m_{1}+m_{2}-1}{m_{2}} \alpha_{1} .
$$


Proof. To prove the first statement, suppose $p<\alpha_{1}+\alpha_{2}$. Suppose further that there exists a $\pi$ such that 0 percolates in $A_{p}$ along $\pi$. We will show that this leads to a contradiction.

For this, assume that $\pi_{1}(0)=\alpha_{2}$, the proof in case $\pi_{1}(0)=\alpha_{1}$ is analogous. It is clear that the corresponding $\tilde{\pi}$ only can be of the following form:

$$
\tilde{\pi}=(e_{2}, \underbrace{-e_{1}, \ldots,-e_{1}}_{r_{1} \text { elements }}, e_{2}, \underbrace{-e_{1}, \ldots,-e_{1}}_{r_{2} \text { elements }}, e_{2}, \ldots),
$$

where $r_{1}+\cdots+r_{n}=\left\lfloor n \alpha_{2} / \alpha_{1}\right\rfloor$, for all $n \geq 1$. But $\left\{n \alpha_{2}\left(\bmod \alpha_{1}\right) \mid n \in \mathbb{N}\right\}$ is dense in $\left[0, \alpha_{1}\right]$, so

$$
\sup _{n}\left[n \alpha_{2}-\left(r_{1}+\cdots+r_{n}\right) \alpha_{1}\right]-\inf _{n}\left[n \alpha_{2}-\left(r_{1}+\cdots+r_{n}\right) \alpha_{1}\right]=\alpha_{1} .
$$

This implies that $\sup _{n}\left[\pi_{n}(0)\right]-\inf _{n}\left[\pi_{n}(0)\right]=\alpha_{1}+\alpha_{2}$ which contradicts our hypothesis. From this argument it also immediately follows that inf $\left\{p \mid 0 \in \mathscr{P}_{p}\right\}=$ $\alpha_{1}+\alpha_{2}$, proving the first statement by an application of Lemma 4.1.

To prove the second statement, take $\tilde{\pi}$ as above. It is easy to check that $\pi_{n}(0) \in\left\{\left(i / m_{2}\right) \alpha_{1}, \quad i=0, \ldots, m_{1}+m_{2}-1\right\}, \quad$ for all $n \in \mathbb{N}$. This proves $p_{c} \leq\left[\left(m_{1}+m_{2}-1\right) / m_{2}\right] \alpha_{1}$.

For the reverse inequality, suppose that there exists a $\pi \in \prod_{2}$ such that $\pi_{i}(0)=\pi_{j}(0)$ for some $i, j$ such that $|i-j| \leq m_{1}+m_{2}-1$. This implies that there are $r, s \in \mathbb{Z}$ such that $|r|+|s|<m_{1}+m_{2}$ and $r \alpha_{1}+s \alpha_{2}=0$. This contradicts our choice of $m_{1}$ and $m_{2}$ and it follows that $p_{c} \geq\left[\left(m_{1}+m_{2}-1\right) / m_{2}\right] \alpha_{1}$.

Observe that if we replace 0 by any other point in $\left[0, \alpha_{1}+\alpha_{2}\right]$, respectively $\left\{\left(i / m_{2}\right) \alpha_{1}, i=0, \ldots, m_{1}+m_{2}-1\right\}$, the same construction also works and that these are the only points for which this is true. This implies that $\mathscr{P}_{p_{\mathrm{c}}}$ is either the whole interval $A_{p_{c}}$ or a finite set, depending on the ratio of $\alpha_{1}$ and $\alpha_{2}$ being irrational or not. Now we will turn our attention to the higher-dimensional case. Here it will become clear why we first treated the two-dimensional case. For a treatment of the higher-dimensional case we define the following map, whose significance will become clear later on.

Definition 4.6. Let $\mathcal{O}_{d}$ be the set

$$
\left\{\left(x_{1}, \ldots, x_{d}\right) \in \mathbb{R}^{d} \mid 0 \leq x_{1} \leq \cdots \leq x_{d}\right\} .
$$

Define a map $M_{d}$ from $\mathcal{O}_{d}$ into itself by $M_{d}\left(\left(x_{1}, \ldots, x_{d}\right)\right)=\left(y_{1}, \ldots, y_{d}\right)$, where $y_{1}, y_{2}, \ldots, y_{d}$ are the numbers $x_{1}, x_{2}-x_{1}, \ldots, x_{d}-x_{1}$ arranged in increasing order.

We first establish the following elementary property concerning the iterates of $M_{d}$.

Lemma 4.7. Let $x=\left(x_{1}, \ldots, x_{d}\right) \in \mathcal{O}_{d}$. Write $t_{n}$ for $\left(M_{d}^{n}(x)\right)_{1}$ and $s_{n}$ for $\left(M_{d}^{n}(x)\right)_{2}$. Then $\lim _{n \rightarrow \infty} t_{n}=0$. Furthermore, if $t_{n} \neq 0$ for all $n$ then $\lim _{n \rightarrow \infty} s_{n}=0$.

Proof. It is easy to see that $t_{n}$ and $s_{n}$ are both non-increasing, so the limits exist. Suppose $\lim _{n \rightarrow \infty} t_{n}=\varepsilon$ for some $\varepsilon>0$. Then we subtract at least $\varepsilon$ from all the other coefficients at each iteration. This implies that $t_{n}<\varepsilon$ for $n$ sufficiently large. To prove the second statement, suppose $s_{n} \geq \varepsilon$ for all $n$. Take $n$ large such that $0<t_{n}<\varepsilon$. Now at each iteration we subtract at least $t_{n}$ from $s_{n}$ until $s_{n}<\varepsilon$. This proves the lemma. 
If $d \geq 3$, we remark that the third smallest coefficient does not tend to zero under general conditions, as one might think at first sight.

Furthermore, we need the following concept of induced paths. Consider interval percolation with parameters $0<\alpha_{1} \leq \cdots \leq \alpha_{d}$. It follows from Theorem 4.5 that $p_{c}\left(\alpha_{1}, \ldots, \alpha_{d}\right) \leq \alpha_{1}+\alpha_{2}$. Checking the proof of Theorem 4.5 , we observe that if 0 percolates in $A_{p}$, then it also percolates along some $\pi$ such that $0 \leq \pi_{n}(0)<\alpha_{1}+\alpha_{2}$ for all $n$. We may therefore assume that if 0 percolates along $\pi$, it has this last property. In the following we only consider paths $\pi$ without immediate reversals, i.e. $\tilde{\pi}_{n+1} \neq-\tilde{\pi}_{n}$ for all $n$. Any path $\pi$ in $\mathbb{Z}^{d}$ such that $\pi_{n}(0) \geq 0$ for all $n$, has the property that $\pi_{n}(0) \geq \alpha_{1}$ infinitely often. Therefore it makes sense to define

$$
\begin{gathered}
n_{1}=n_{1}(\pi)=\min \left\{n \mid \pi_{n}(0) \geq \alpha_{1}\right\}, \\
n_{k+1}=n_{k+1}(\pi)=\min \left\{n>n_{k} \mid \pi_{n}(0) \geq \alpha_{1}\right\},
\end{gathered}
$$

for all $k>1$. Note that $n_{k+1}-n_{k} \leq 2$ for all $k$.

Now consider the sequence

$$
\left(\pi_{n_{0}}(0):=\alpha_{1}, \pi_{n_{1}}(0), \pi_{n_{2}}(0), \ldots\right) .
$$

It follows by construction that $\pi_{n_{k}}(0) \in\left[\alpha_{1}, \alpha_{1}+\alpha_{2}\right)$, for all $i$. Furthermore, we have $\pi_{n_{k+1}}(0)-\pi_{n_{k}}(0) \in\left\{ \pm \alpha_{1}, \pm\left(\alpha_{2}-\alpha_{1}\right), \ldots, \pm\left(\alpha_{d}-\alpha_{1}\right), \pm\left(\alpha_{j}-\alpha_{i}\right), 2 \leq i<j\right\}$. (There might be indices $i, j, n, m$ such that $\alpha_{i}-\alpha_{j}=\alpha_{n}-\alpha_{m}$, but we only write $\pi_{n_{k+1}}(0)-\pi_{n_{k}}(0)=\alpha_{i}-\alpha_{j}$ if $\pi_{n_{k+1}}-\pi_{n_{k}}=e_{i}-e_{j}$.)

Starting from the sequence above, we construct a new sequence as follows:

If $\pi_{n_{k+1}}(0)-\pi_{n_{k}}(0)=\left(\alpha_{j}-\alpha_{i}\right)$, for $i$ and $j$ larger than 1 , then we insert an element in this sequence between $\pi_{n_{k}}(0)$ and $\pi_{n_{k+1}}(0)$ with value $\pi_{n_{k}}(0)-\left(\alpha_{i}-\alpha_{1}\right)=$ $\pi_{n_{k+1}}(0)-\left(\alpha_{j}-\alpha_{1}\right)$, which is greater than or equal to $\alpha_{1}$. The sequence obtained this way is denoted by $\pi^{\prime}\left(\alpha_{1}\right)=\left(\pi_{0}^{\prime}\left(\alpha_{1}\right), \pi_{1}^{\prime}\left(\alpha_{1}\right), \pi_{2}^{\prime}\left(\alpha_{1}\right), \ldots\right)$. It has the property that

$$
\pi_{n+1}^{\prime}\left(\alpha_{1}\right)-\pi_{n}^{\prime}\left(\alpha_{1}\right) \in\left\{ \pm \alpha_{1}, \pm\left(\alpha_{2}-\alpha_{1}\right), \ldots, \pm\left(\alpha_{d}-\alpha_{1}\right)\right\}
$$

for all $n$. It is not difficult to see that $\sup _{n}\left(\pi_{n}(0)\right)=\sup _{n}\left(\pi_{n}^{\prime}\left(\alpha_{1}\right)\right)$. We can identify $\pi^{\prime}\left(\alpha_{1}\right)$ with a path $\pi^{\prime}$ in $\mathbb{Z}^{d}$ in a natural way as follows: $\pi_{0}^{\prime}:=0$; if $\pi_{n}^{\prime}=z$ for some $n \geq 0$, and

(i) $\pi_{n+1}^{\prime}\left(\alpha_{1}\right)-\pi_{n}^{\prime}\left(\alpha_{1}\right)= \pm \alpha_{1}$, then $\pi_{n+1}^{\prime}:=z \pm e_{1}$.

(ii) $\pi_{n+1}^{\prime}\left(\alpha_{1}\right)-\pi_{n}^{\prime}\left(\alpha_{1}\right)= \pm\left(\alpha_{j}-\alpha_{1}\right)$, then $\pi_{n+1}^{\prime}:=z \pm e_{j}$, for $j=2, \ldots, d$.

We say that $\pi^{\prime}$ is induced by $\pi$. If we consider interval percolation with parameters $\alpha_{1}, \alpha_{2}-\alpha_{1}, \ldots, \alpha_{d}-\alpha_{1}$, which are not necessarily in increasing order, then $\pi_{n}^{\prime}\left(\alpha_{1}\right)$ is the image of $\alpha_{1}$ under the map $\pi_{n}^{\prime}: \mathbb{R} \rightarrow \mathbb{R}$, and this is in agreement with our usual notation.

For example, let $\left(\alpha_{1}, \alpha_{2}, \alpha_{3}\right)=(4,7,10)$ and

$$
\left(\pi_{0}(0), \pi_{1}(0), \ldots\right)=(0,7,3,10,0,7,3,10,0, \ldots) .
$$

Then

$$
\left(\pi_{0}^{\prime}\left(\alpha_{1}\right), \pi_{1}^{\prime}\left(\alpha_{1}\right), \ldots\right)=(4,7,10,4,7,10,4, \ldots),
$$

and the path $\pi^{\prime}$ is given by

$$
\left(\pi_{0}^{\prime}, \pi_{1}^{\prime}, \pi_{2}^{\prime}, \ldots\right)=\left(0, e_{2}, 2 e_{2}, 2 e_{2}-e_{3}, 3 e_{2}-e_{3}, \ldots\right) .
$$


This construction can be continued. We can define the induced path of $\pi^{\prime}$ etc. We say that $\pi^{\prime}$ is a first level induced path, the induced path of $\pi^{\prime}$ is a second level induced path and so on.

Now we can state the next theorem, which deals with the critical value in the higher-dimensional case.

THEOREM 4.8. Consider interval percolation with parameters $0<\alpha_{1} \leq \cdots \leq \alpha_{d}$. Denote $\left(\alpha_{1}, \ldots, \alpha_{d}\right)$ by $x^{0}=\left(x_{1}^{0}, \ldots, x_{d}^{0}\right)$ and define $x^{i}=M_{d}\left(x^{i-1}\right)$, for $i>0$, where $M_{d}$ is defined as in Definition 4.6. Then we have

(i) $p_{c}\left(\alpha_{1}, \ldots, \alpha_{d}\right)=\sum_{i=0}^{\infty} x_{1}^{i}$.

(ii) Let $d>2$. If, for some $i_{0} \in \mathbb{N}$ and some $2 \leq n<d$

$$
\begin{gathered}
\frac{1}{n-1}\left(x_{1}^{i_{0}}+\cdots+x_{n}^{i_{0}}\right) \leq x_{n+1}^{i_{0}}, \text { then } \\
p_{c}\left(\alpha_{1}, \ldots, \alpha_{d}\right)=\sum_{i=0}^{i_{0}-1} x_{1}^{i}+p_{c}\left(x_{1}^{i_{0}}, \ldots, x_{n}^{i_{0}}\right) .
\end{gathered}
$$

(iii) Let $d>2$. If $(*)$ does not hold for any $i_{0}$ and $2 \leq n<d$ then

$$
p_{c}\left(\alpha_{1}, \ldots, \alpha_{d}\right)=\frac{1}{d-1}\left(\alpha_{1}+\cdots+\alpha_{d}\right) \text {. }
$$

Proof. (i) First note that $\lim _{i \rightarrow \infty} p_{c}\left(x^{i}\right)=0$. To see this, observe that if $x_{1}^{i}=0$ for some $i$ we trivially have $p_{c}\left(x^{j}\right)=0$, for all $j \geq i$. If $x_{1}^{i} \neq 0$ for all $i \in \mathbb{N}$ we have that $\lim _{i \rightarrow \infty} p_{c}\left(x^{i}\right) \leq \lim _{i \rightarrow \infty}\left(x_{1}^{i}+x_{2}^{i}\right)=0$, by Theorem 4.5 and Lemma 4.7. To prove (i), it therefore suffices to prove the following

$$
p_{c}\left(x^{i}\right)=x_{1}^{i}+p_{c}\left(x^{i+1}\right), \text { for all } i \geq 0 .
$$

We prove $(* *)$ for $i=0$, using the concept of induced paths. We transform the problem of interval percolation with parameters $x_{1}^{0}, \ldots, x_{d}^{0}$, and which takes place in the interval $\left[0, \alpha_{1}+\alpha_{2}\right)$, in an interval percolation problem with parameters $x_{1}^{1}, \ldots, x_{d}^{1}$, which takes place in the interval $\left[\alpha_{1}, \alpha_{1}+\alpha_{2}\right)$.

Suppose 0 percolates in $[0, p]$ along $\pi$. The induced path of $\pi$ is denoted by $\pi^{\prime}$. We may assume that $0 \leq \pi_{n}(0)<\alpha_{1}+\alpha_{2}$ for all $n$. This means that for the induced path $\pi^{\prime}$ we have $\alpha_{1} \leq \pi_{n}^{\prime}\left(\alpha_{1}\right)<\alpha_{1}+x_{1}^{1}+x_{2}^{1} \leq \alpha_{1}+\alpha_{2}$.

We first prove ' $\geq$ ' in (**). It is enough to show that the induced path $\pi^{\prime}$ of $\pi$ has the property that $d\left(\pi_{n}^{\prime}, 0\right)$ is not bounded, where $d$ denotes Euclidean distance in $\mathbb{Z}^{d}$. Once we have shown this, we remove possible circuits in $\pi^{\prime}$ to obtain a self-avoiding path along which $\alpha_{1}$ percolates in $\left[\alpha_{1}, p\right]$. Then ' $\geq$ ' follows by an application of Lemma 4.1 (iii) where 0 is replaced by $\alpha_{1}$.

It follows from the construction of induced paths that for $2 \leq i \leq d$, each step $\pm e_{i}$ in $\pi$ gives rise to exactly one step $\pm e_{i}$ in $\pi^{\prime}$ and vice versa. The order in which steps of $+e_{i}$ and $-e_{i}$ appear is the same in both paths. Now let, for all $n \geq 0$,

$$
\begin{aligned}
& u_{i}(n)=\operatorname{card}\left\{j \leq n \mid \tilde{\pi}_{j}=e_{i}\right\}, \\
& v_{i}(n)=\operatorname{card}\left\{j \leq n \mid \tilde{\pi}_{j}=-e_{i}\right\}, \\
& w_{i}(n)=u_{i}(n)-v_{i}(n),
\end{aligned}
$$


and let $w_{i}^{\prime}(n)$ be defined analogously for the path $\pi^{\prime}$. Note that $w_{i}(n)$ and $w_{i}^{\prime}(n)$ are just the $i$ th coordinate of $\pi_{n}$ and $\pi_{n}^{\prime}$ respectively. If $w_{i}(n)$ is bounded for all $i$, then $\pi_{n}$ does not tend to infinity, so that is at least one index $i_{0}$ say, such that $w_{i_{0}}(n)$ is unbounded. Suppose that this is the only index for which this is true. Then it is not difficult to see that $\pi_{n}(0)$ is unbounded. But $0 \leq \pi_{n}(0) \leq p$ and hence this is a contradiction. We conclude that there are at least two indices $i_{0}$ and $j_{0}$ say, such that $w_{i_{0}}(n)$ and $w_{j_{0}}(n)$ are unbounded. At least one of these indices must be larger than $1, j_{0}$ say. It follows from the observation above that in that case, $w_{j_{0}}^{\prime}(n)$ is also unbounded. Hence $d\left(\pi_{n}^{\prime}, 0\right)$ is unbounded and we have proved ' $\geq$ '.

The reverse inequality is proved in the same spirit: Suppose $\alpha_{1}$ percolates along $\pi^{\prime}$ in $\left[\alpha_{1}, p\right]$ (with parameters $x_{1}^{1}, \ldots, x_{d}^{1}$ ), where $\alpha_{1} \leq p \leq \alpha_{1}+\alpha_{2}$. We may assume that $\alpha_{1} \leq \pi_{n}^{\prime}\left(\alpha_{1}\right)<\alpha_{1}+\alpha_{2}$ for all $n$. Consider a path $\pi$ which induces $\pi^{\prime}$. Such a $\pi$ exists trivially and because $\pi^{\prime}$ is self-avoiding we can take $\pi$ such that it has no immediate reversals. We now follow the argument as above, with $\pi$ and $\pi^{\prime}$ interchanged. This proves the reverse inequality.

(iii) If (*) does not hold it is easy to see that $\lim _{i \rightarrow \infty} x_{j}^{i}=0$, for all $j=1, \ldots, d$. (Consider the smallest index $n+1$ for which the limit is not zero to derive a contradiction.) Assuming that (*) does not hold, let $y(i)=\sum_{n=1}^{d} x_{n}^{i}$. It is clear from the construction that $y(i)-y(i+1)=(d-1) x_{1}^{i}$. This implies that

$$
\sum_{i=0}^{\infty} x_{1}^{i}=\frac{1}{d-1} \sum_{i=0}^{\infty}\left(y(i)-y(i+1)=\frac{1}{d-1} y(0)=\frac{1}{d-1}\left(\alpha_{1}+\cdots+\alpha_{d}\right),\right.
$$

proving (iii). Note that it follows that

$$
\frac{1}{d-1}\left(\alpha_{1}+\cdots+\alpha_{d}\right)
$$

is an upper bound for the critical value in interval percolation with parameters $\alpha_{1} \leq \cdots \leq \alpha_{d}$.

(ii) If (*) does hold, we have by (iii) that

$$
\begin{aligned}
p_{c}\left(x_{1}^{i_{0}}, \ldots, x_{n}^{i_{0}}\right) & \leq \frac{\left(x_{1}^{i_{0}}+\cdots+x_{n}^{i_{0}}\right)}{(n-1)} \\
& \leq x_{n+1}^{i_{0}} .
\end{aligned}
$$

Now suppose $p_{c}\left(x_{1}^{i_{0}}, \ldots, x_{d}^{i_{0}}\right)<p_{c}\left(x_{1}^{i_{0}}, \ldots, x_{n}^{i_{0}}\right)$. Suppose $\pi \in \prod_{d}$ is such that 0 percolates along $\pi$ in $\left[0, p_{c}\left(x_{1}^{i_{0}}, \ldots, x_{d}^{i_{0}}\right)\right]$. Because there is strict inequality, at least one of the extra parameters on the l.h.s. must be used in $\pi$. But this is not possible because

$$
p_{c}\left(x_{1}^{i_{0}}, \ldots, x_{d}^{i_{0}}\right)<x_{n+1}^{i_{0}} \text {. So } p_{c}\left(x_{1}^{i_{0}}, \ldots, x_{d}^{i_{0}}\right)=p_{c}\left(x_{1}^{i_{0}}, \ldots, x_{n}^{i_{0}}\right)
$$

and (ii) follows.

The preceding theorem states that, starting with $d$ parameters, we can either reduce the dimension or calculate the critical value directly. If we reach dimension 2 we can calculate the critical value according to Theorem 4.5. The question of course is if it is possible to decide whether or not $(*)$ holds. For this, first consider the case in which $\alpha_{1}, \ldots, \alpha_{d}$ are all rational, say $\alpha_{1}=a_{1} / c, \ldots, \alpha_{d}=a_{d} / c$. It is clear from the construction that $x_{1}^{i+1}+x_{2}^{i+1} \leq x_{1}^{i}+x_{2}^{i}-1 / c$ if $x_{1}^{i} \neq 0$. But this implies that 
$x_{1}^{i}=0$ for $i$ sufficiently large and $p_{c}\left(\alpha_{1}, \ldots, \alpha_{d}\right)$ can be written as a finite sum. Of course (*) holds if $x_{1}^{i_{0}}=0$ as is seen by taking $n=2$.

For example, let $\alpha_{1}=\frac{9}{25}, \alpha_{2}=\frac{13}{25}, \alpha_{3}=\frac{15}{25}$. Then $x^{0}=\left(\frac{9}{25}, \frac{13}{25}, \frac{15}{25}\right), x^{1}=\left(\frac{4}{25}, \frac{6}{25}, \frac{9}{25}\right)$, $x^{2}=\left(\frac{2}{25}, \frac{4}{25}, \frac{5}{25}\right), x^{3}=\left(\frac{2}{25}, \frac{2}{25}, \frac{3}{25}\right), x^{4}=\left(0, \frac{1}{25}, \frac{2}{25}\right)$. This implies that $p_{c}\left(\frac{9}{25}, \frac{13}{25}, \frac{15}{25}\right)=$ $\frac{9+4+2+2}{25}=\frac{17}{25}$.

To study $(*)$ in some detail, we show that if $(*)$ holds for some $i_{0}$ and $n$, it also holds for $i$ and $n$ for every $i>i_{0}$. To see this, suppose first that $x_{1}^{i_{0}} \leq x_{n+1}^{i_{0}}-x_{1}^{i_{0}}$. Then

$$
\frac{1}{n-1}\left(x_{1}^{i_{0}+1}+\cdots+x_{n}^{i_{0}+1}\right)=\frac{1}{n-1}\left(x_{1}^{i_{0}}+\cdots+x_{n}^{i_{0}}-(n-1) x_{1}^{i_{0}}\right)
$$

which is smaller than or equal to $x_{n+1}^{i_{0}}-x_{1}^{i_{0}}=x_{n+1}^{i_{0}+1}$.

If $x_{1}^{i_{0}}>x_{n+1}^{i_{0}}-x_{1}^{i_{0}}$, then

$$
\frac{1}{n-1}\left(x_{1}^{i_{0}+1}+\cdots+x_{n}^{i_{0}+1}\right)=\frac{1}{n-1}\left(x_{1}^{i_{0}}+\cdots+x_{n+1}^{i_{0}}-(n+1) x_{1}^{i_{0}}\right)
$$

which is smaller than or equal to $x_{n+1}^{i_{0}}-x_{1}^{i_{0}} \leq x_{n+1}^{i_{0}+1}$.

This means that the set where

$$
\frac{1}{n-1}\left(x_{1}^{i}+\cdots+x_{n}^{i}\right) \leq x_{n+1}^{i}
$$

for some $i$ and $n$ is invariant under $M_{d} .(*)$ holds if we eventually enter such an invariant set.

Now let $d>2$ and define

$$
\Gamma:=\left\{x^{0} \in \mathcal{O}_{d} \mid(*) \text { does not hold for any } 2 \leq n<d \text { and } i_{0} \in \mathbb{N}\right\} .
$$

If $x \in \Gamma$, the whole line through the origin and $x$ is in $\Gamma$, because the condition $(*)$ is homogeneous. Points on the same line through the origin are in fact equivalent in interval percolation. Therefore, we consider the set $\Gamma^{\prime} \subset \mathbb{R}^{d-1}$ which is obtained from $\Gamma$ as follows:

$$
\Gamma^{\prime}=\left\{\left(\frac{x_{1}}{x_{d}}, \ldots, \frac{x_{d-1}}{x_{d}}\right) \mid\left(x_{1}, \ldots, x_{d}\right) \in \Gamma\right\} .
$$

We now believe that the following is true.

CONJECTURE 4.9. $\Gamma^{\prime}$ has Lebesgue-measure zero.

The conjecture roughly says that if we choose parameters randomly, according to normalized Lebesgue-measure on $\Gamma^{\prime}$, with probability one we can calculate the critical value in a finite number of calculations. In ([MN]), we shall prove that the conjecture is true if $d=3$.

5. The percolation functions $\Theta^{I}$ and $\Theta^{C}$

In this section we will show how to determine the percolation functions in circle and interval percolation. The most important step in the analysis of $\Theta^{I}$ and $\Theta^{C}$ is the following lemma.

LeMMA 5.1. Consider interval percolation. Define, for all $\omega \geq 0, \rho_{w}:=$ $\sup \left\{\eta \leq \omega \mid \eta \in \mathscr{P}_{p_{c}}\right\}$. Then $p_{c, \omega}=p_{c}+\omega-\rho_{\omega}$. In the case of circle percolation we have an analogous result. 
Proof. First observe that for $\omega \in \mathscr{P}_{p_{c}}$ there is nothing to prove. Suppose $\omega \notin \mathscr{P}_{p_{c}}$. According to Proposition 2.7, $\rho_{\omega} \in \mathscr{P}_{p_{c}}$. Suppose $\rho_{\omega}$ percolates in $A_{p_{c}}$ along $\pi$. This implies that $\pi_{n}(\omega) \in\left[\omega-\rho_{\omega}, p_{c}+\omega-\rho_{\omega}\right]$ and we have $p_{c, \omega} \leq p_{c}+\omega-\rho_{\omega}$.

To prove the reverse inequality, suppose $p_{c, \omega}<p_{c}+\omega-\rho_{\omega}$. Suppose further that $\omega$ percolates in $A_{p_{c, \omega}}$ along $\pi$. Now consider the first index $n_{0}$ such that $\pi_{n_{0}}(\omega) \notin\left[p_{c, \omega}-p_{c}, p_{c}\right]$ and suppose $\pi_{n_{0}} \omega>p_{c}$. Let $r_{0}=\pi_{n_{0}}(\omega)$. Of course, $r_{0} \leq p_{c, \omega}$. Let $\pi^{\prime \prime}$ be such that $p_{c}$ percolates in $A_{p_{c}}$ along $\pi^{\prime \prime}$. Consider the $n_{0}$-concatenation $\pi^{*}$, say, of $\pi$ and $\pi^{\prime \prime}$. It then follows that $r_{0}-p_{c} \leq \pi_{n}^{*}(\omega) \leq r_{0}$, for all $n$. This implies that

$0 \leq \pi_{n}^{*}\left(\omega-\left(r_{0}-p_{c}\right)\right) \leq p_{c}$ which means that $\omega-\left(r_{0}-p_{c}\right) \in \mathscr{P}_{p_{\mathrm{c}}}$. But $\omega-\left(r_{0}-p_{c}\right)>\rho_{\omega}$ so this contradicts our choice of $\rho_{\omega}$. If $\pi_{n_{0}}(\omega)<p_{c, \omega}-p_{c}$, the arguments are analogous, using that 0 percolates in $A_{p_{c}}$. This implies that $p_{c, \omega} \geq p_{c}+\omega-\rho_{\omega}$.

It follows from Lemma 5.1 that $\Theta$ is completely determined by the set $\mathscr{P}_{p_{c}}$. Therefore we will analyze this set in detail. We first establish the following lemma whose easy verification is left to the reader.

Lemma 5.2. Consider interval percolation. Suppose $\eta \in A_{p}$ and $\omega \in \mathscr{P}_{p}$ for some $p$. Suppose further that there exists an element $\pi^{l} \in \prod_{d}^{l}$ for some $l$, such that starting in $\eta$ and making steps in accordance with $\pi^{l}$, we stay in $A_{p}$ and end up at $\omega$. Then $\eta \in \mathscr{P}_{p}$.

From now on we suppose that there exists an index $i_{0}$ such that

$$
p_{c}\left(\alpha_{1}, \ldots, \alpha_{d}\right)=\sum_{i=0}^{i_{0}-1} x_{1}^{i}+p_{c}\left(x_{1}^{i_{0}}, x_{2}^{i_{0}}\right) .
$$

Define $y_{0}=0, y_{j}:=\sum_{i=0}^{j-1} x_{1}^{i}, 0<j \leq i_{0}$ and let $B_{i}:=\mathscr{P}_{p_{c}} \cap\left[y_{i}, p_{c}\right]$ for $i=0, \ldots, i_{0}$.

The proof of Theorem 4.8 shows that $\omega \in\left[y_{i}, p_{c}\right]$ percolates in $\left[0, p_{c}\right]$ along some $\pi$ iff $\omega$ percolates in $\left[y_{i}, p_{c}\right]$ along some $i$ th level induced path. The parameters associated with this induced path are $x_{1}^{i}, \ldots, x_{d}^{i}$. The remark following Theorem 4.5 now implies that $B_{i_{0}}$ is known, because on $\left[y_{i_{0}}, p_{c}\right]$ we have a two-parameter system by assumption. $B_{i_{0}}$ is either the whole interval $\left[y_{i_{0}}, p_{c}\right]$ or a finite set, depending on the ratio of $x_{1}^{i_{0}}$ and $x_{2}^{i_{0}}$ being irrational or not. The following lemma tells us how to determine $\mathscr{P}_{\boldsymbol{p}_{\mathrm{c}}}$ once $B_{\boldsymbol{i}_{0}}$ is known.

Lemma 5.3. Suppose $B_{i_{0}}$ is known as above. Then

$$
B_{i}=B_{i+1} \cup\left\{B_{i+1}-x_{1}^{i}\right\}, i=0, \ldots, i_{0}-1,
$$

where, for a subset $B$ of $\mathbb{R},\{B-x\}=\{y \in \mathbb{R} \mid y=b-x$ for some $b \in B\}$.

Proof. Suppose $\omega \in B_{i} \backslash B_{i+1}$. Then $\omega$ percolates along an ith level induced path in $\left[y_{i}, p_{c}\right]$, with parameters $x_{1}^{i}, \ldots, x_{d}^{i}$. But $w-x_{1}^{i} \notin\left[y_{i}, p_{c}\right]$. So for $\omega$ to percolate, $\omega+x_{1}^{i}$ must be smaller than or equal to $p_{c}$. According to Lemma 5.2, $\omega+x_{1}^{i} \in \mathscr{P}_{p_{c}}$ and thus $\omega+x_{1}^{i} \in B_{i+1}$. This implies that $\omega \in B_{i+1}-x_{1}^{i}$. For the other inclusion, suppose $\omega \in B_{i+1}$. Then of course, $\omega \in B_{i}$. If $\omega \in\left\{B_{i+1}-x_{1}^{i}\right\} \backslash B_{i+1}$, we have that $\omega+x_{1}^{i} \in B_{i+1}$ and $\omega \in\left[y_{i}, p_{c}\right]$. So $\omega+x_{1}^{i}$ percolates and, according to Lemma 5.2, so does $\omega$ which implies that $\omega \in B_{i}$.

It follows from this lemma that the set $\mathscr{P}_{p_{c}}$ is either a finite set or a finite union of closed intervals. In general, we do not know how to determine $\mathscr{P}_{p_{c}}$ in case the 
condition we imposed does not hold. In ([MN]) we shall prove that in the case of interval percolation with parameters $0 \leq \alpha_{1} \leq \alpha_{2} \leq \alpha_{3}$, the set $\mathscr{P}_{p_{\mathrm{c}}}$ is the whole interval $\left[0, p_{c}\right]$ if the condition does not hold.

Resuming the results of this section, we now state the following theorem which gives the precise form of the percolation functions $\Theta^{I}$ and $\Theta^{C}$.

THEOREM 5.4. Consider interval percolation with parameters $0<\alpha_{1} \leq \cdots \leq \alpha_{d}$. Then

(i) $\Theta(p)=\mu\left(T^{p-p_{c}}\left(\mathscr{P}_{p_{c}}\right)\right)$, for $p \geq p_{c}$, where $T^{\varepsilon}(B)=\left\{x+\varepsilon^{\prime} \mid x \in B, 0 \leq \varepsilon^{\prime} \leq \varepsilon\right\}$, for every set $B \subset \mathbb{R}$ and $\varepsilon>0$.

(ii) Suppose the condition stated after Lemma 5.2 holds. Then $\Theta^{I}$ is piecewise linear and concave on $\left[p_{c}, \infty\right)$ and $\Theta^{I}$ is continuous iff $\mu\left(\mathscr{P}_{p_{c}}\right)=0$.

(iii) If $\alpha_{d} \leq \frac{1}{2}$ then the percolation function $\Theta^{C}$ can be written as

$$
\Theta^{C}\left(\alpha_{1}, \ldots, \alpha_{d}\right)(p)=\Theta^{I}\left(\alpha_{1}, \ldots, \alpha_{d}, 1-\alpha_{d}\right)(p),
$$

for $p<1$.

Proof. The theorem is an immediate consequence of the preceding and Theorem 4.4.

Examples. (A) Consider interval percolation in $\mathbb{Z}^{2}$, with two parameters $0<\alpha_{1} \leq \alpha_{2}$. We already noticed that $\mathscr{P}_{p_{c}}$ is either the whole interval $\left[0, p_{c}\right]$ or the set $\left\{\left(i / m_{2}\right) \alpha_{1}, i=0, \ldots, m_{1}+m_{2}-1\right\}$, where $m_{1}$ and $m_{2}$ are as in Theorem 4.5. Consequently, $\Theta^{I}\left(\alpha_{1}, \alpha_{2}\right)$ is of the form as in figure 1 .
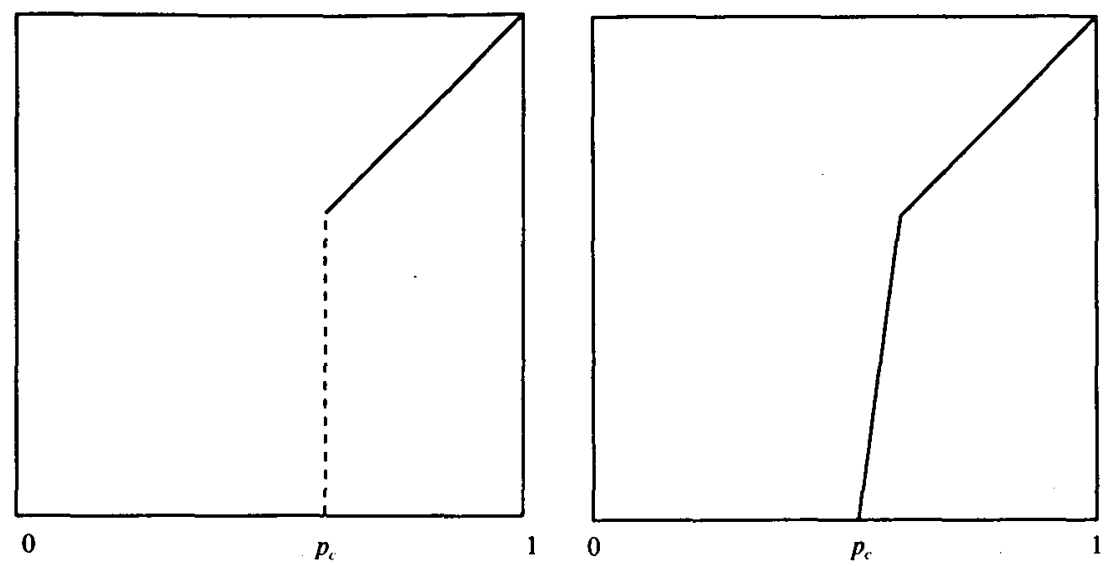

FIGURE 1. $\Theta^{\prime}\left(\alpha_{1}, \alpha_{2}\right)$ for $\alpha_{1} \notin \alpha_{2} \notin \mathbf{Q}$ resp. $\alpha_{1} / \alpha_{2} \in \mathbf{Q}$.

(B) Circle percolation with parameters $\alpha_{1}=\frac{7}{20}, \alpha_{2}=\frac{9}{20}$. It follows from Theorem 4.9 and Lemma 5.3 that $p_{c}^{C}=\frac{11}{20}$ and $\mathscr{P}_{p_{\mathrm{C}}}^{C_{\mathrm{C}}}=\left\{0, \frac{2}{20}, \frac{4}{20}, \frac{7}{20}, \frac{9}{20}, \frac{11}{20}\right\}$. This implies that $\Theta^{C}$ is as in figure $2(\mathrm{i})$.

(C) Circle percolation with parameters $\alpha_{1}=3 \pi / 21, \alpha_{2}=\frac{10}{21}$. Now (*) holds for $i_{0}=1$ and the critical value is $(21-3 \pi) / 21$. According to Lemma 5.3, the set $\mathscr{P}_{p_{c}}^{C_{c}}$ is

$$
\left[0, \frac{21-6 \pi}{21}\right] \cup\left[\frac{3 \pi}{21}, \frac{21-3 \pi}{21}\right] \text {. }
$$

The percolation function is as in figure 2 (ii). 

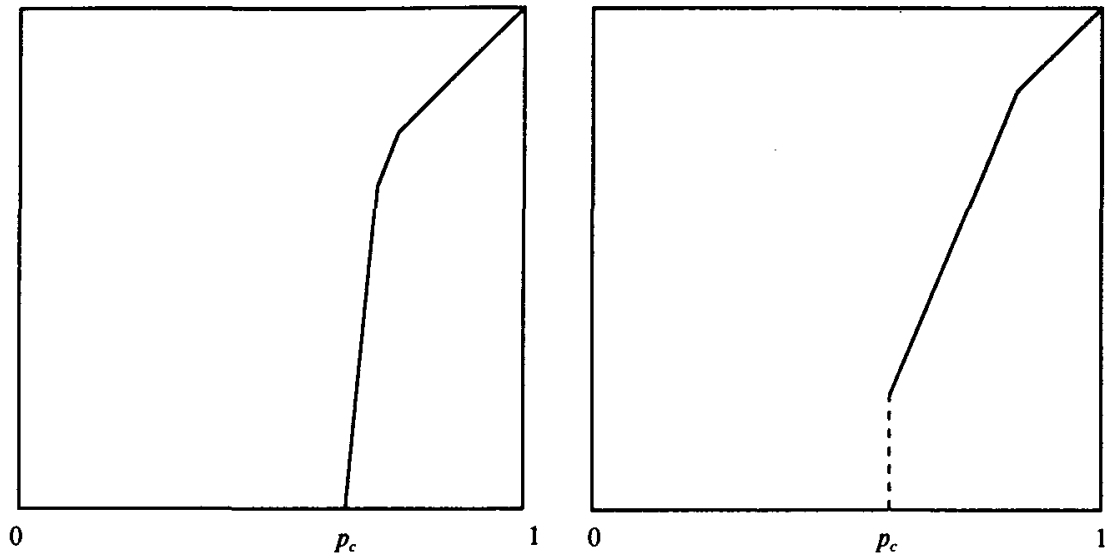

Figure 2. The percolation function for examples $B$ and $C$ resp.

Acknowledgements. I would like to thank the referee for finding a mistake in an earlier version of the manuscript. Furthermore, I would like to thank Alberto Gandolfi for his remarks and suggestions to improve the paper.

\section{REFERENCES}

[B] J. v.d. Berg. Percolation theory on pairs of matching lattices. J. Math. Phys. 22(1) (1981), 152-157.

[C] J. P. Conze. Entropie d'un groupe abelien de transformations. $Z$. Wahrscheinlichkeitstheorie und verw. Geb. 25 (1972), 1-30.

[Ka] H. Kesten. Percolation Theory for Mathematicians. Birkhauser: Boston, 1982.

[Kb] H. Kesten. Scaling relations for 2D-percolation. Commun. Math. Phys. 109 (1987), 109-156.

[Kc] H. Kesten. A scaling relation at criticality for 2D-percolation. Percolation Theory and Ergodic Theory of Infinite Particle Systems, pp. 203-212, ed. H. Kesten, Springer: New York, 1987.

[MN] R. W. J. Meester \& T. Nowicki. Infinite clusters and critical values in two-dimensional circle percolation. Israel J. Math. (1989) submitted for publication.

[T] B. Toth. A lower bound for the critical probability of the square lattice site percolation. Z. Wahrscheinlichkeitstheorie und verw. Geb. 69 (1985), 19-22. 